\title{
Inhalt des 16. Bandes
}

\section{Heft $1 / 2$}

Kleinert, H. M., Group Dynamics of Elementary Particles

\section{Heft 3}

Horstmann, M., Einfluß der thermischen Gitterschwingungen auf die Streuung schneller

Elektronen in Kristallen . . . . . . . . . . . . . . . . . . . . . . . . 75

JEGERLEHNER, F., Zeitrichtung und Kosmologie . . . . . . . . . . . . . . . . . . 137

\section{Heft 4}

Rotrer, I., Clustereigenschaften leichter Kerne . . . . . . . . . . . . . . . . . . 195

\section{Heft 5}

Risken, H., Zur Statistik des Laserlichts . . . . . . . . . . . . . . . . . . . . 261

FAessler, A., Surface Delta Interaction in Heavy Deformed Nuclei . . . . . . . . 309

\section{Heft 6}

Fedorov, V. V., and A. Pazman, Design of Physical Experiments (Statistical Methods) 325 SCHRÖDER, U. E., Noether's Theorem and the Conservation Laws in Classical Field

Theories . . . . . . . . . . . . . . . . . . . . . . 357

\section{Heft 7}

Enflo, B. O., and B. E. Latrent, Dirac Formalism for Arbitrary Spin in S-Matrix Theory

\section{Heft 8}

Krebs, K. H., Electron Ejection from Solids by Atomic Particles with Kinetic Energy 419

\section{Heft 9}

Hegerfeldt, G. C., and J. Hennig, Coupling of Space-Time and Internal Symmetry . 491

\section{Heft 10}

Dadtcourt, G., and G. Wallis, The Cosmic Blackbody Radiation . . . . . . . . . . 545

\section{Heft 11/12}

Baranov, P. S., L. V. Fil'kov and G. A. Sokol, Proton Compton Effect . . . . . . . 595

Hamprecht, B., and H. M. Kleinert, Decays of Baryon Resonances in an $O$ (3.1) Model . . . . . . . . . . . . . . . . . . . . . . . . . 635

Kaschudr, F., Compositeness Conditions for Particle Poles in Partial Wave Scattering Amplitudes I . . . . . . . . . . . . . . . . . . . . . . . . . . . . . . . . 669

REEH, H., Symmetry Operations and Spontaneously Broken Symmetries in Relativistic Quantum Field Theories . . . . . . . . . . . . . . . . . . . . 687

MrCHEJDA, L., On Distinguishing between Peripheral and Central High Energy Collisions 707 Barashenkov, V.S., and G. M. ZinovJev, Unitary-Symmetrical Theory of Multiple Particle Production 
Herausgeber: Prof. Dr. Frank Kaschluhn, Prof. Dr. Artur Losche, Prof. Dr. Rudolf Ritschl und Prof. Dr. Robert Rompe; Manuskripte sind zu richten an die Schriftleitung: Dr. Lutz Rothkirch, Sektion Physik der Humboldt-Universität zu Berlin, 104 Berlin, Hessische Str. 2. Verlag: Akademie-Verlag GmbH, 108 Berlin, Leipziger Str. 3/4, Fernruf: 220441, Telex-Nr. 0112020, Postscheckkonto: Berlin 35021. Die Zeitschrift „Fortschritte der Physik" erscheint monatlich; Bezugspreis eines Heftes 8,- (Sonderpreis for die DDR 6,- M). Bestellnummer dieses Bandes: 1027/16. - Satz und Druck: VEB Druckhaus „Maxim Gorki“, 74 Altenburg. Bez. Leipzlg, Carl-von-Ossietzky-Str. 30/31. - Veröffentlicht unter der Lizenznummer 1324 des Presseamtes beim Vorsitzenden des Ministerrates der Deutschen Demokratischen Republik. 


\section{FORTSCHRITTE DER}

PHYSIK

IIERACSGEGEBEA IU ALFTRAGE DER PHYSIKALISCHEX GISLLLSCHAFT 1. DER

DECTSCHEN DEMOK R.ITISCHEN REPUBLIK

Vo. F. KASCHLLIIN, A. LÖSCHE, R. RITSCHL L.T R. ROMPE:

BAND $16 \cdot$ HEFT $1 / 2 \cdot 1968$

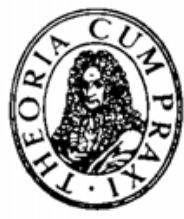

A K A D E M I E - V E R L A G - B E R L I N 


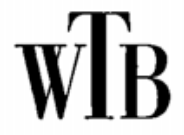

WISSENSCHAFTLICHE TASCHENBÜCHER

REIHE MATHEMATIK - PHYSIK

Prof. Dr. Harry Pfeifer

Elektronik für den Physiker

I: Theorie linearer Bauelemente

1966. 187 Seiten -55 Abbildungen -11 Tabellen $-8^{\circ}-$ M 8,-

II: Die Elektronenröhre

1966. 100 Seiten -28 Abbildungen -9 Tabellen $-8^{\circ}-$ M 8,

III: Schaltungen mit Elektronenröhren

1966. 170 Seiten -67 Abbildungen -3 Tabellen $-8^{\circ}-$ M 8, -

IV: Leitungen und Antennen

1967. 200 Seiten -59 Abbildungen -5 Tabellen $-8^{\circ}-$ M 8, -

V: Mikrowellenelektronik

1967. 131 Seiten -42 Abbildungen -3 Tabellen $-8^{\circ}-$ M 8,

VI: Halbleiterelektronik

1967. 217 Seiten -81 Abbildungen -19 Tabellen $-8^{\circ}-$ M 8,-

Bestellungen durch eine Buchhandlung erbeten

Ausführlicher Prospekt der gesamten Reihe auf Wunsch vom

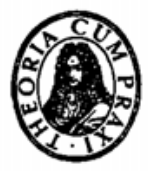

AKA DE M I E-VER LAG - B E R L I N

Die „FORTSCHRITTE DER PHYSIK“ sind durch den Buchhandel zu beziehen.

Falls keine Bezugsmöglichkeit durch eine Buchhandlung vorhanden ist, wenden Sie sich bitte in der Deutschen Demokratischen Republik

an den AKADEMIE-VERLAG, GmbH, 108 Berlin, Leipziger Straße 3-4

in der Deutschen Bundesrepublik

an die Auslieferungsstelle: KUNST UND WISSEN, Inhaber Erich Bieber, 7 Stuttgart, Wilhelmstraße 4-6

bei Wohnsitz im übrigen Ausland an den Deutschen Buch-Export und -Import, GmbH, 701 Leipzig, Postschließfach 276 oder direkt an den AKADEMIE-VERLAG, GmbH, 108 Berlin, Leipziger Straße 3-4 